\title{
Study on the intercalation of fullerene derivate into ordered phases liquid crystalline polymer
}

\author{
Chen-An Wang, ${ }^{1,2}$ Jrjeng Ruan ${ }^{2}$ and U-Ser Jeng ${ }^{1,3}$ \\ ${ }^{1}$ National Synchrotron Radiation Research Center, Hsinchu Science Park, Hsinchu 30076, Taiwan, \\ ${ }^{2}$ Department of Materials Science and Engineering, National Cheng Kung University, Tainan City 701401, Taiwan, \\ ${ }^{3}$ Department of Chemical Engineering, National Tsing Hua University, Hsinchu 30013, Taiwan \\ wang.ca@nsrrc.org.tw
}

The phase separation relationship between polymer and fullerene have been treated as a major influence that impact the operation of bulk heterojunction solar cells. In this research, we investigate the intercalation behavior between PBTTT-C14(PBTTT (Poly[2,5bis(3-tetradecylthiophen-2-yl)thieno[3,2-b]thiophene])) and PCBM ([6,6]-Phenyl C71 butyric acid methyl ester, PC71BM) to unveil the phase separation relationship in this system.

Temperature dominates the competition between the intercalation of PCBM into PBTTT-C14 order phase and self-crystallization of PCBM. At lower temperature, PCBM tends to intercalate into the cavity among PBTTT-C14 side chain. However, at higher temperature PCBM tends to crystallization.

In the other hand, PCBM are not able to intercalate into PBTTT-C14 domain once the side chain regularity is reduced by partially soluble solvent on PBTTT-C14. No self-assembling relationship are found between PBTTT-C14 and PCBM either at high or low temperature.

Keywords: PBTTT, intercalation, composite liquid crystals 